\title{
IDENTIFICATION AND CHARACTERIZATION OF PSWRKY1 INVOLVED IN THE ABIOTIC STRESS RESPONSES OF POLYGONATUM SIBIRICUM (SOLOMON'S SEAL)
}

\author{
YU, S. H. - YE, J. F. ${ }^{*}-$ FAN, J. G. ${ }^{*}-$ MA, D. J. - ZHENG, Y. - BU, P. T. \\ Forestry Biotechnology and Analysis Test Center, Liaoning Academy of Forestry Sciences, \\ Shenyang 110032, China \\ ${ }^{*}$ Corresponding authors \\ e-mail: yejingfeng527@163.com (Ye, J. F.); fanjungang178@163.com (Fan, J. G.) \\ (Received $16^{\text {th }}$ Jul 2021; accepted $28^{\text {th }}$ Oct 2021)
}

\begin{abstract}
WRKY protein is an important transcription factor in response to abiotic stress in plants. However, a systematic identification and characterization of WRKY genes has not been carried out for the medicinal plant Polygonatum sibiricum that has a strong ability to resist various abiotic stresses. In this study, we isolated a novel WRKY gene from P. sibiricum and compared its sequence structure with other plants. PsWRKYI possesses two typical WRKY domains and two $\mathrm{C}_{2} \mathrm{H}_{2}$ zinc-finger motifs. Evolutionary analysis indicated that PsWRKYI is most closely related to the WRKY protein from Eucalyptus grandis. Expression analysis showed that expression levels of PsWRKYI were induced by cold and drought stresses but not salt stress. Overexpression of PsWRKYI in Arabidopsis improved the seed germination and growth conditions of transgenic plants under drought and cold stresses. Furthermore, SOD activity and proline content in transgenic plants were higher than those in WT under cold and drought stresses, whereas MDA levels and relative electrolyte leakage in transgenic plants were lower than those in WT under same stresses. These results indicated that PsWRKYI improved the tolerance to cold and drought stresses. This study is significant for understanding the molecular mechanism behind $P$. sibiricum cold and drought stresses tolerance.
\end{abstract}

Keywords: WRKY transcription factors, medicinal plant, cold stress, drought stress, overexpression

Abbreviations: TF, Transcription factor; $\mathrm{H}_{2} \mathrm{O}_{2}$, hydrogen peroxide; TCM, Traditional Chinese Medicine; ABA, Abscisic acid; SOD, Super oxide dismutase; MDA, Malondialdehyde; NJ, Neighbor-joining; PCR, Polymerase chain reaction; qRT-PCR, Quantitative real time polymerase chain reaction; NBT, Nitro blue tetrazolium; WT, Wild type; NLS, Nuclear localization signal; POD, Peroxidase; CAT, Catalase; ROS, Reactive oxygen species; $P O D$, Peroxidase; TBA, thiobarbituric acid

\section{Introduction}

Plants are easily exposed to a great variety of abiotic and biotic stresses, including extreme changes of temperature, light, drought, and pathogens etc. The main limiting environmental factors influencing plant cultivation worldwide are abiotic stresses, especially low temperature and drought stress. To endure these stresses, plants have evolved comprehensive reprogramming of the cellular metabolism (Rushton et al., 2012). A key step in the responses to all kinds of stresses is transcription factor (TF), which regulates transcriptional regulation of many downstream target genes (Mitsuda and Ohme-Takagi, 2009). WRKY protein is an important family of transcription factors, which are named after highly conserved WRKYGQK motifs. WRKY members include three groups (I, II and III) and various subgroups (e.g. IIa, IIb, etc.) based on the number of WRKY domains and the type of zinc finger motifs. WRKY TFs play an essential role in resistance of biotic or abiotic stresses in plants (Jiang et al., 2017; Eulgem et al., 2000). Many WRKY genes have been isolated in various plant species. For example, 72 WRKY genes have been identified in Arabidopsis, 55 in cucumber, 104 in poplar, 109 in rice, 83 in tomato,79 in potato, 46 in 
rapeseed, and 119 in corn (Eulgem et al., 2000; Amorim et al., 2017; Ng et al., 2018; Liu et al., 2019).

Some WRKY TFs are closely related to tolerance of abiotic stresses for plant such as cold, salt, drought, and heat etc. Four banana fruit WRKY TFs are involved in ABA (abscisic acid)-induced cold tolerance by increasing ABA levels (Luo et al., 2017). FcWRKY70 is a WRKY gene from Fortunella crassifolia, which confers drought tolerance by modulating putrescine synthesis (Gong et al., 2015). MuWRKY3 from Arachis hypogaea can enhance drought resistance by accumulating less malondialdehyde and hydrogen peroxide $\left(\mathrm{H}_{2} \mathrm{O}_{2}\right)$ (Kiranmai et al., 2018). PbrWRKY53 plays a positive role in drought resistance by promoting production of vitamin $\mathrm{C}$ via regulating PbrNCEDl expression in Pyrus betulaefolia. GsWRKY20 from soybean improves drought stress resistance in transgenic soybean (Luo et al., 2013). VaWRKY12 and VaWRKY33 from grapevine both improve the cold tolerance of transgenic Arabidopsis and grapevine calli (Zhang et al., 2019; Sun et al., 2019). CsWRKY46 from cucumber enhances cold tolerance in transgenic plants and positively regulates the ABA-dependent cold signaling pathway (Zhang et al., 2016). Although some WRKY genes have been studied in many plants, the functions of most of WRKY genes are still poorly understood, especially in many non-model plants.

Polygonatum species which belong to the family Asparagaceae are widely distributed throughout the temperate Northern Hemisphere with 71 species. Due to their positive effect on human health, these plants have been used in Traditional Chinese Medicine (TCM). $P$. sibiricum is known as 'Huangjing' (Solomon's seal), which distributes in the northern hemisphere, mainly from southwest China to Japan (Pan et al., 2020). Huangjing can treat some disease such as lung disorders, osteoporosis, fatigue, and feebleness. More particularly, Huangjing has stronger tonic effect than other Polygonatum species, tonifying the spleens and kidneys (Jo et al., 2017; Zhao et al., 2018). Because $P$. sibiricum distributes mostly in thickets, woodlands or hillsides, it can resist various abiotic stresses such as drought, cold, and water etc. (Liu et al., 2009; Qu et al., 2010; Zhao et al., 2018). Although P. sibiricum has a strong ability to resist abiotic stresses, the genes related to abiotic stresses resistance from $P$. sibiricum are still poorly identified.

In this study, we cloned a novel WRKY gene PsWRKYI in $P$. sibiricum and analyzed its sequence structure and evolution relationship. Expression analysis indicated expressing pattern of PsWRKY1 under drought, cold, and salt stress. Overexpression of PsWRKY1 was generated to evaluate its function during drought, cold, and salt stresses. The growth conditions of transgenic Arabidopsis under drought, cold, and salt stresses were identified. SOD activity, proline content, relative electrolyte leakage, and MDA levels were analyzed in transgenic Arabidopsis to evaluate function of PsWRKY1 for resisting abiotic stresses.

\section{Materials and methods}

\section{Plant materials and treatments}

Seedlings of $P$. sibiricum, which were originally collected from Qingyuan county in Fushun city (Liaoning Province, China), were transplanted in plastic boxes filled with soil substrates and placed in a controlled growth chamber with the temperature set at $25^{\circ} \mathrm{C}$, a 16 $\mathrm{h}$ light $/ 8 \mathrm{~h}$ dark photoperiod, and a humidity of $45 \%$. Three-week-old plantlets with three well developed leaves were used for different treatments. For the cold treatments, whole plantlets grown in conical flasks were placed at $4{ }^{\circ} \mathrm{C}$ in an illuminated incubation chamber (16 h light/8 h dark, 8000 Lux), and fresh leaves were harvested at 0, 2, 6, ,12, 24, $48 \mathrm{~h}$, and $72 \mathrm{~h}$ time points. For dehydration treatment, seedlings were treated with 20\%PEG 6000 for 
$0,2,6,12,24,48 \mathrm{~h}$, and $72 \mathrm{~h}$. For salt stress, the seedlings were treated with $200 \mathrm{mM} \mathrm{NaCl}$ solution for $0,1,3,6,12$ and $24 \mathrm{~h}$. For each treatment, at least 15 seedlings were used and the leaves, stems and roots were sampled from three randomly collected seedlings at designated time point and frozen immediately in liquid nitrogen and stored at $-80{ }^{\circ} \mathrm{C}$ until use. Three biological replicates were collected for each sample.

\section{RNA extraction, gene isolation and bioinformatic analysis}

Total RNA of from the collected samples was extracted by TaKaRa MiniBEST Plant RNA Extraction Kit (TaKaRa, Japan) according to the manufacturer's instruction. First strand cDNA synthesis was performed using PrimeScriptTM RT reagent Kit with gDNA Eraser (Takara), and stored at $-20^{\circ} \mathrm{C}$ until use. To isolate WRKY gene from $P$. sibiricum, homologous sequences from other species in the Genbank were aligned. A set of primers for gene amplification were designed by Primer 5 software (http://www.Premierbiosoft.com) (Table S1). The conserved fragment of WRKY gene was amplified from cDNA and several clones were sequenced. To obtain the whole sequence of WRKY gene, 5'-RACE and 3'RACE experiments were performed using a Full RACE Kit (TaKaRa) after the acquisition of the conserved sequence. To validate the whole coding sequence of WRKY genes, primers were designed on the basis of the assembled results, and several clones were sequenced to correct errors introduced during PCR. Multiple sequence alignments were done using $\begin{array}{lllll}\text { DNAMAN } & 8 & \text { (http://www.lynnon.com) } & \text { and } & \text { MEGA }\end{array}$ (http://www.megasoftware.net/mega.php). Conserved motifs were identified using MEME Suite 4.10.1 (http://meme-suite.org/) and WebLogo 3.4 (http://weblogo.threeplusone.com/). To compare evolutionary relationship of WRKY genes in plants, the phylogenetic trees were constructed by MEGA 5.10 (http://www.megasoftware.net/mega.php) based on the neighbor-joining (NJ) method and bootstrap analysis (1,000 replicates). Highly similar homologous genes were downloaded from NCBI (http://www.ncbi.nlm.nih.gov/) and presented in Table $S 2$.

\section{Vector constructs and plant transformation for overexpression}

The PCR products of WRKY, carried by vector pMD-18-T, were cloned into vector pCAMBIA 1301. In this vector, the enhanced CaMV 35S promoter drives the constitutive expression of transgenes. The expression vectors were transformed into Agrobacterium tumefaciens EHA105 by the freeze-thaw method (Zhang et al., 2016). The foreign genes were then transfected into wild-type Arabidopsis thaliana using leaf disc transformation (Kiranmai et al., 2018). The resulting seeds were screened on 1/2 MS medium containing $50 \mathrm{mg} \cdot \mathrm{L}^{-1}$ hygromycin (HPT), and the presence of the transgene in $\mathrm{T} 0$ and $\mathrm{T} 1$ plants was confirmed by PCR amplification of the HPT gene on genomic DNA and by analyzing the expression of $W R K Y$ from cDNA T3 transgenic plants were subjected to screening. Initially, PCR was carried out to identify the presence of the target transgenes in the transgenic plants.

\section{Expression analysis of WRKY genes}

To detect the expression of WRKY gene under abiotic stress, qRT-PCR was performed using $10 \mu \mathrm{L}$ of FastStart Universal SYBR Green Master (Aidlab, Beijing) on a StepOne Plus real-time PCR instrument (Aidlab, Beijing). Each sample was prepared with three biological and three technical replicates, and the relative expression was calculated using the relative quantification method (2- $\Delta \Delta \mathrm{CT}$ ). $\beta$-actin (GenBank accession no. EC969944) was used as a reference gene for $P$. sibiricum samples. 


\section{Relative electrolyte leakage test}

After treatment of $4^{\circ} \mathrm{C}$ cold stress, $200 \mathrm{mM} \mathrm{NaCl}, 20 \% \mathrm{PEG}$ for $12 \mathrm{~h}$, respectively, three T3 transgenic plants for WRKY and WT plants were immediately sampled, and 10 leaf discs of each sample were obtained to determine relative electrolyte leakage. Leaf discs were immersed in deionized water for $10 \mathrm{~h}$ at ambient temperature. The initial electrical conductivity $\left(\mathrm{S}_{1}\right)$ was measured using a DDS-IIA detector (Shanghai, China), and then the final electrical conductivity $\left(\mathrm{S}_{2}\right)$ was measured after boiling for $10 \mathrm{~min}$. Relative electrolyte leakage (L) was calculated as:

$$
\mathrm{L}(\%)=\mathrm{S}_{1} \div \mathrm{S}_{2} \times 100
$$

Nine replications were used for all treatments.

\section{Estimation of malondialdehyde (MDA) contents}

MDA contents were analyzed using the thiobarbituric acid method (Del-Rio et al., 2005). Briefly, after treatment mentioned above, three T3 transgenic plants for WRKY and WT plants were accurately weighed with the leaves $(0.5 \mathrm{~g})$ to be tested, and was added with precooled phosphate buffer $(1 \mathrm{ml})$, then grinded, diluted with buffer solution to $5 \mathrm{ml}$. After centrifuging the solution at $1000 \mathrm{r} / \mathrm{min}$ for $20 \mathrm{~min}$, the supernatant solution $(2 \mathrm{ml})$ was added and mixed with TBA $(2 \mathrm{ml})$. The reaction mixture was boiled for $30 \mathrm{~min}$ and then cooled down to room temperature. Lastly, the absorbance was noted at 532 and $600 \mathrm{~nm}$. MDA concentration was calculated using the extinction coefficient of $155 \mathrm{~m} \mathrm{M}^{-1} \mathrm{~cm}^{-1}$ and expressed as $1 \mu \mathrm{mol} \mathrm{g}^{-1}$ dry weight. Nine replications were used for all treatments.

\section{Superoxide dismutase (SOD) activity assay}

SOD activity was assayed by monitoring the inhibition of photochemical reduction of nitro blue tetrazolium (NBT). After treatment mentioned above, the $3 \mathrm{ml}$ reaction mixture contained $2.4 \mathrm{ml}$ of $50 \mathrm{mM}$ buffered phosphate solution ( $\mathrm{pH} 7.8$ ), $0.2 \mathrm{ml}$ of $195 \mathrm{mM}$ methionine, $0.1 \mathrm{ml}$ of $3 \mu \mathrm{M}$ EDTA, $0.2 \mathrm{ml}$ of $1.125 \mathrm{mM} \mathrm{NBT}, 0.1 \mathrm{ml}$ of $60 \mu \mathrm{M}$ riboflavin, and $40 \mu \mathrm{l}$ enzyme extract. The reaction mixtures were illuminated for $20 \mathrm{~min}$ at a light intensity of $300 \mu \mathrm{mol} \cdot \mathrm{m}^{-2} \cdot \mathrm{s}^{-1}$. One unit of SOD activity was defined as the amount of enzyme required to cause $50 \%$ inhibition of the reduction of NBT as monitored at $560 \mathrm{~nm}$.

\section{Determination of proline content}

After treatment mentioned above, leaf materials of T3 transgenic plants and WT plants $(0.5 \mathrm{~g})$ were homogenized in $3 \%(\mathrm{w} / \mathrm{v})$ sulfosalicyclic acid and homogenate filtered through filter paper. After addition of acid ninhydrin and glacial acetic acid, the resulting mixture was heated at $100^{\circ} \mathrm{C}$ for $1 \mathrm{~h}$ in a water bath. The reaction was then stopped by using an ice bath. The mixture was extracted with toluene, and the absorbance of the fraction with toluene extracted from the liquid phase was read at $520 \mathrm{~nm}$.

\section{Statistical analysis}

All data that needed statistical analysis are shown as mean values \pm standard errors of the mean. SPSS Statistics (www.ibm.com/products/spss-statistics) software was used for statistical analysis via Student's $t$-test. Significant differences were considered significant with a probability level of $p<0.05$. 


\section{Results}

\section{Sequence analysis of WRKY gene and evolutionary relationship analysis}

According to sequences of conserved domains in typical WRKY genes, a fragment with 512 bp was amplified by RT-PCR. Using 5'- and 3'-RACE technology, a 751 bp fragment and another $803 \mathrm{bp}$ fragment were obtained, respectively. The mRNA full-length of a WRKY gene was obtained by sequence assembly and re-amplification. This gene is 1,833 bp in length, including an open reading frame (ORF) of 1533 bp that encodes 510 amino acids. This gene was named as PsWRKY1 with an accession No. MK256764 in Genbank. It was showed that PsWRKY1 has the similar typical primary structures to those known WRKY proteins from other plants by comparison of amino acid sequences. All WRKY amino acid sequences all contain two typical WRKY domains that includes the highly conserved amino acid sequence 'WRKYGQK'. Two $\mathrm{C}_{2} \mathrm{H}_{2}$ zinc-finger motifs were adjacent to each WRKY domains, respectively. A conserved nuclear localization signal (NLS) with amino acid sequence KKKV was found at 227-230 amino acid region of PsWRKY1 protein. In addition, we found some differences from each other by some insertions, substitutions and deletions of some amino acid residues in all WRKY proteins (Fig. 1).

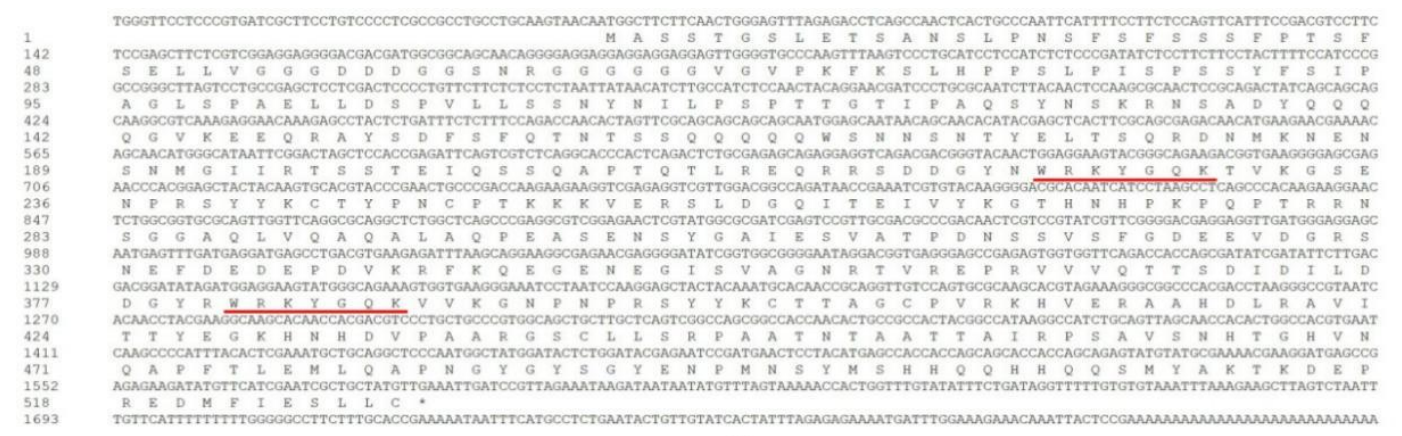

a
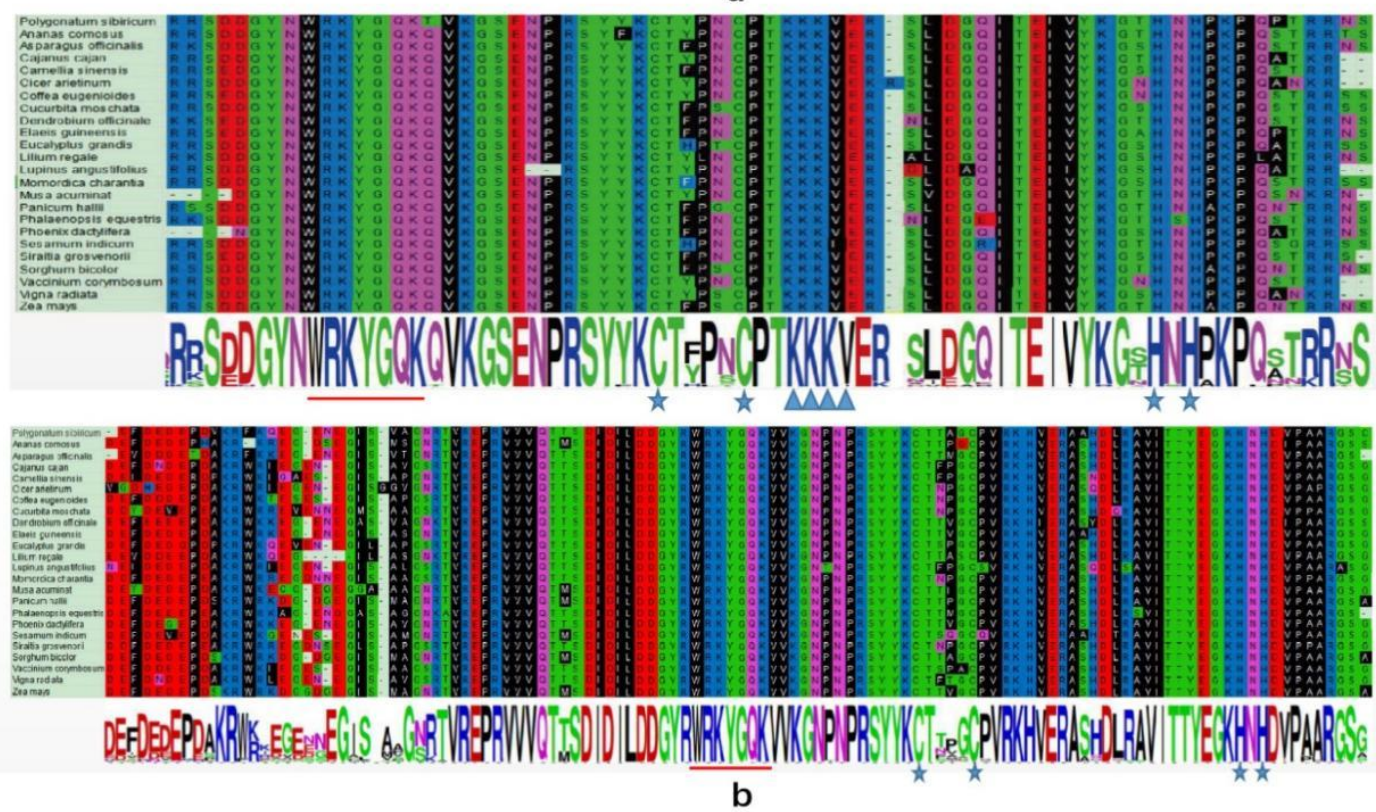

Figure 1. Nucleotide and deduced amino-acid sequences of PsWRKY1 (a) and sequence alignment of PsWRKY1 with several typical WRKY proteins (b). The conserved WRKY domains are marked by red lines. $C$ and $H$ residues in the zinc-finger motifs are marked by asterisks. The conserved nuclear localization signal (NLS) is marked by triangles. GenBank accession number is MK256764 
To understand evolutionary relationship of WRKY proteins, we constructed a phylogenetic tree using the PsWRKY1 and 23 WRKY proteins from other plants. It was showed that all WRKY TFs could be classified into six major groups, and PsWRKY1 was clustered into a monophyletic group with five TFs from Eucalyptus grandis, Sesamum indicum, Ananas comosu, Panicum hallii, and Sorghum bicolor in Group I. PsWRKY1 is most closely related to WRKY protein from Eucalyptus grandiss (Fig. 2).

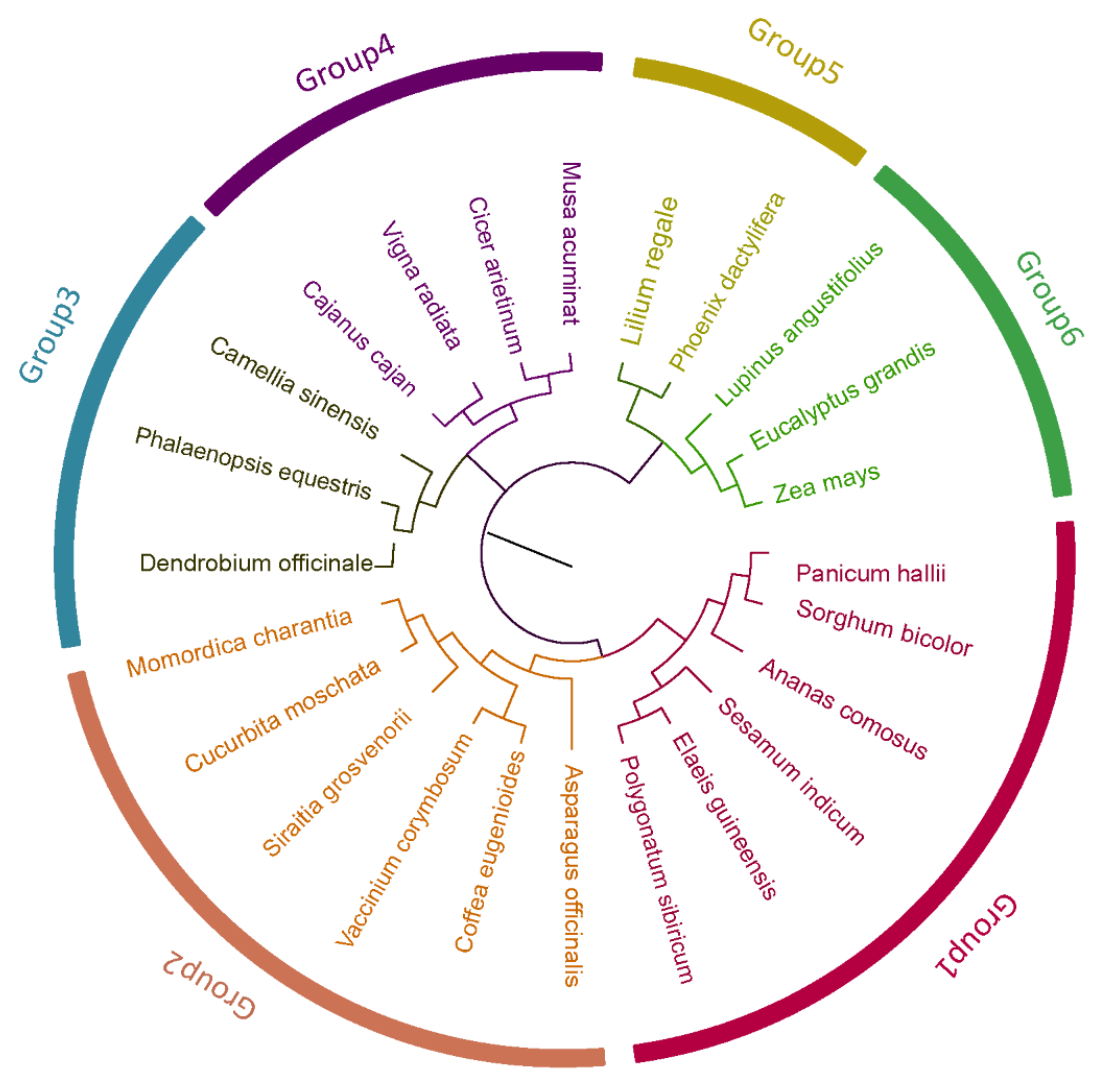

Figure 2. Phylogenetic relationship of PsWRKY1 protein and other stress-related WRKY TFs. The trees were constructed via the neighbor-joining method with a poisson correction model and 1000 bootstrap replicates

\section{Expression of Ps WRKY1 during abiotic stresses}

To identify the expression patterns of PsWRKYI under abiotic stresses, the transcript levels of $P S W R K Y 1$ in stems, leaves, and roots were detected during cold, drought, and salt stresses by qRT-PCR. After cold stress, PsWRKY1 was expressed in leaves and stems of $P$. sibiricum, but not in roots. The expression levels of PsWRKY1 in leaves and stems both increased gradually with the increase of time for cold stress, reaching a peak at $12 \mathrm{~h}$, at approximately 10.70-fold and 8.23-fold of the control $(0 \mathrm{~h})$, respectively. The expression level of $P S W R K Y 1$ in leaves is higher than that in stems. For dehydration stress, PsWRKYI was expressed in all detected tissues. The expression levels of PsWRKYI increased gradually with the increase of treatment time, reaching a peak at 2 days (Fig. 3). Compared to leaves and stems, the expression level of PsWRKY1 in roots is highest, at approximately 14-fold of the control $(0 \mathrm{~d})$. However, the expression of PsWRKY1 is not significantly changed under salt stress (data not shown). 


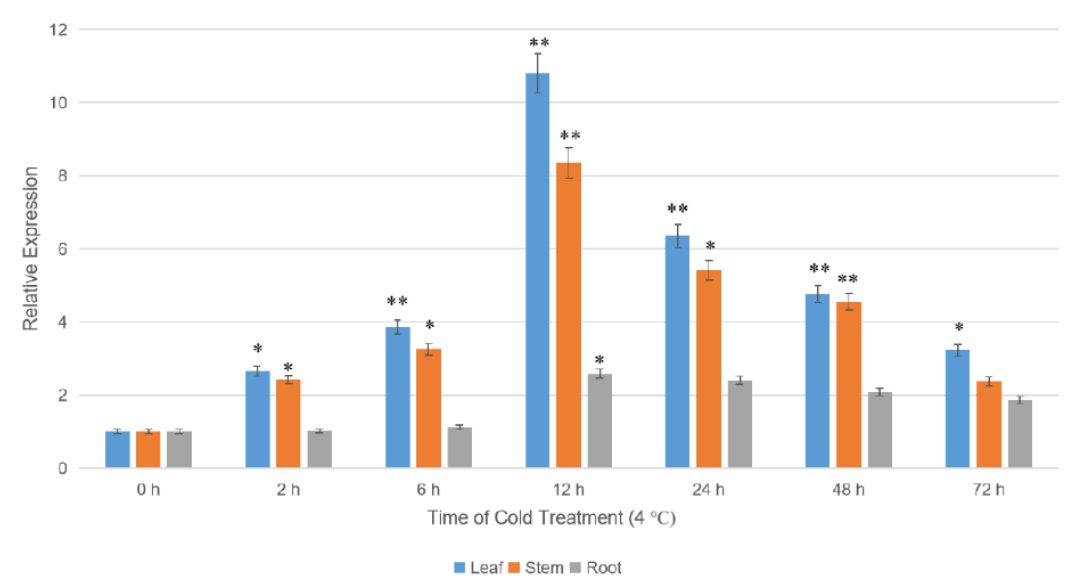

a

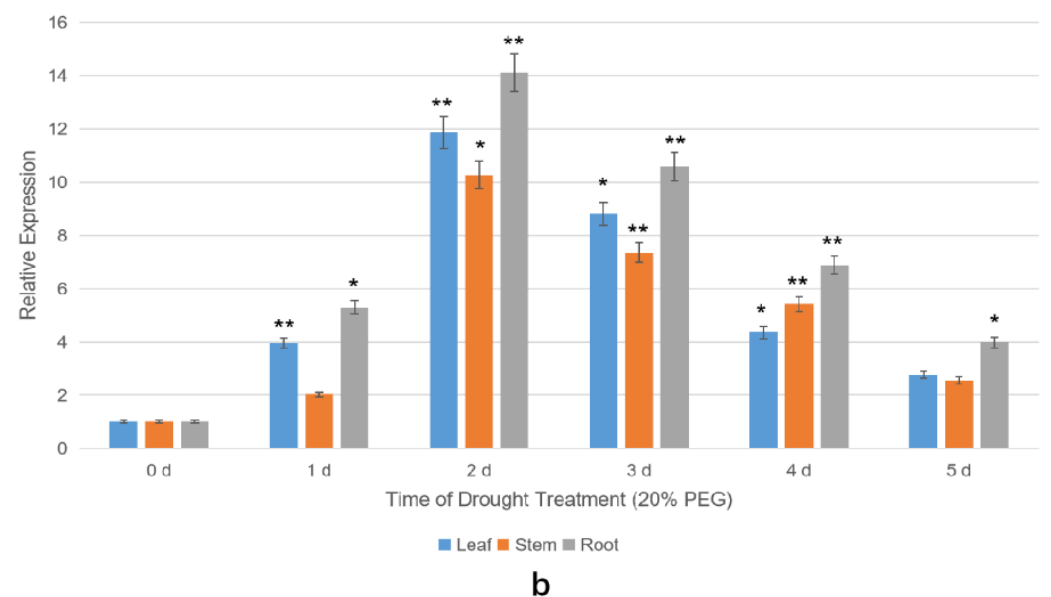

Figure 3. Expression of PsWRKY1 gene under cold stress (a) and drought stress (b). Error bars indicate the standard error; the experiments were repeated three times along with at least three independent repetitions of the biological experiments. Asterisks indicate a significant difference by Student's t-test $(* * P<0.01$ and $* P<0.05)$

\section{Seed germination and seedling growth conditions in transgenic plants under abiotic stresses}

To investigate the main role of PsWRKY1 TFs, an expression construct pCAMBIA 1301-PsWRKY1 was transformed into Arabidopsis thaliana. A homozygous transgenic line was confirmed by qRT-PCR (Fig. S1). Seed germination and growth conditions of wild type (WT) and transgenic plants were tested to evaluate the role of PsWRKY1 under drought and cold stresses, respectively. The germination rate of transgenic plants was higher than that of WT during drought stress, while the growth conditions of transgenic plants were better than those of WT under cold stress (Fig. 4). These results showed that PsWRKY1 might improve the resistance to cold and drought stress in transgenic plants.

\section{Physiological indicators of Ps WRKY1 overexpression plants}

To further identify the role of PsWRKY1, SOD activity, proline content, relative electrolyte leakage, and MDA were tested under cold, drought, and salt stress, respectively. The results indicated that SOD activity and proline content in transgenic plants were all higher than those in WT plants under cold stress and dehydration treatment, 
while MDA levels and relative electrolyte leakage were lower than those in WT plants. However, there are no significant differences in all physiological indicators between the transgenic plants and the WT control under salt stress conditions (Fig. 5). These results showed that PsWRKY1 enhanced the cold and drought tolerances by increasing the SOD activity and proline content, and reducing the relative electrolyte leakage and MDA content in $P$. sibiricum.
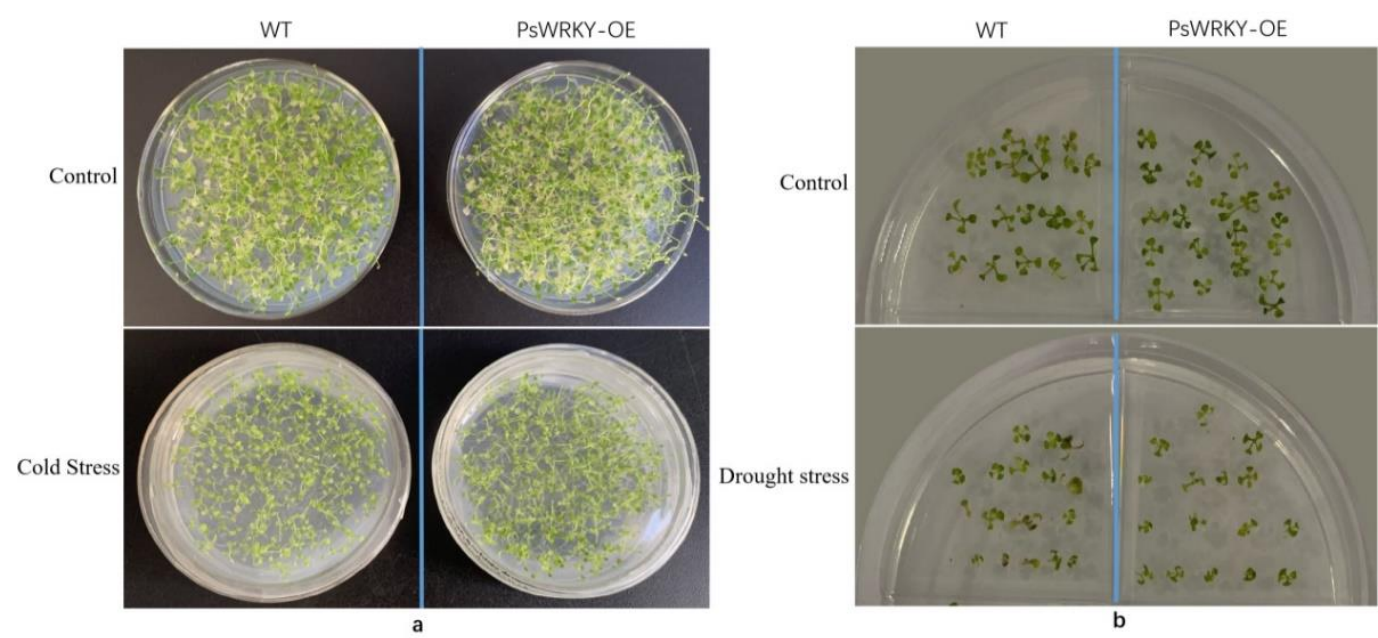

Figure 4. The growth conditions of WT and PSWRKY1 overexpression plants (OE) on MS medium under abiotic stresses. (a) The phenotypes of the three-week-old seedlings on MS medium after cold treatment $\left(4{ }^{\circ} \mathrm{C}\right)$ for 7 days and recovery under normal growth conditions for

7 days. (b) Germination conditions of WT and PSWRKY1 OE plants on MS medium under drought stress with 20\% PEG 6000. WT and OE seeds were vernalized for 3 days and grown on MS medium with $20 \%$ PEG 6000 for 7 days
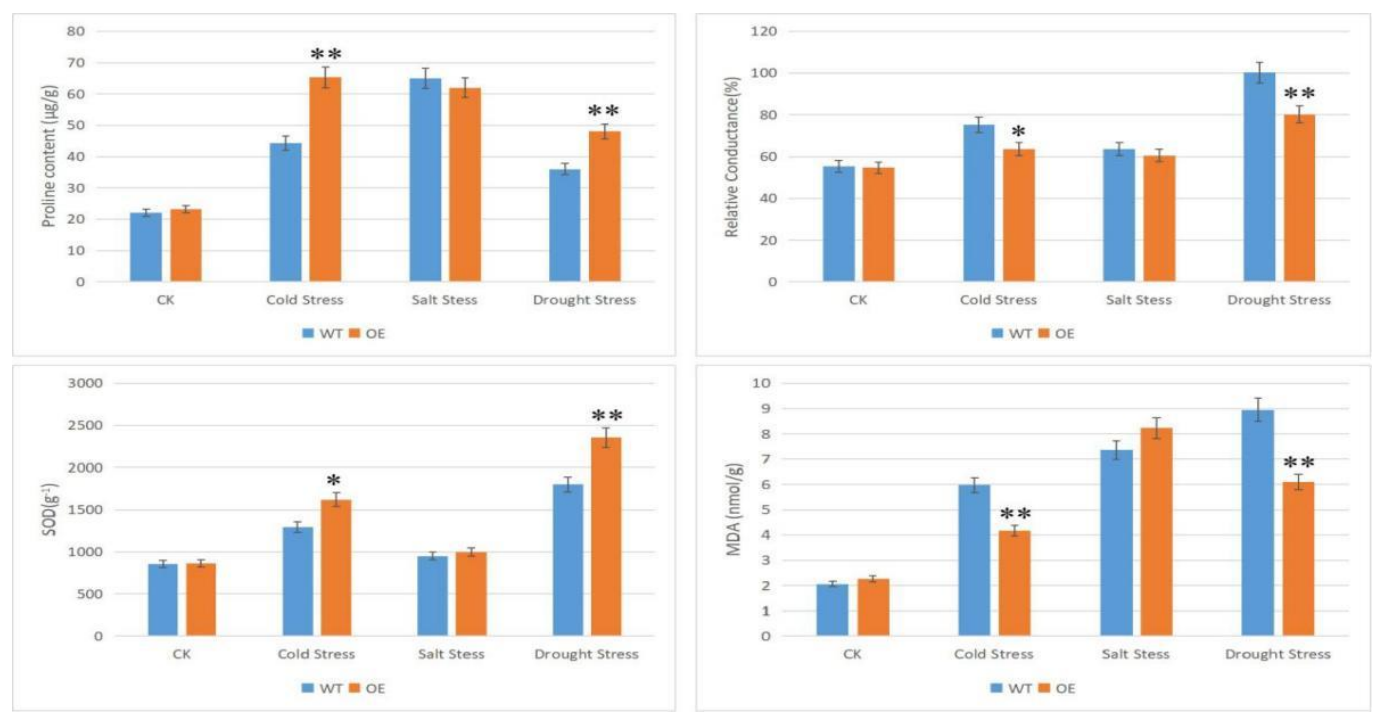

Figure 5. Physiological indexes of PsWRKYI overexpression plants $(O E)$ under abiotic stresses, including the SOD activity, proline content, relative electrolyte leakage, and MDA content under cold stress, salt stress, and drought stress. Error bars represent standard deviations (SDs). All the data represent the means $\pm S D$ s of three independent biological replicates. Asterisks indicate a significant difference compared to WT by Student's t-test

$(* * P<0.01$ and $* P<0.05)$ 


\section{Discussion}

Previous studies indicated WRKY transcription factors play vital roles in medicating the responses to abiotic and biotic stresses. Abiotic stresses including salinity stress, drought stress, cold and heat stress, and oxidative stress etc. adversely affect the development processes in plants (Rushton et al., 2012). Expression of many WRKY genes is induced under various abiotic stresses, improving the tolerance to abiotic stresses in plants. $P$. sibiricum, a kind of medicinal plant, is often used to treat some diseases and tonify the spleens and kidneys. Due to the medicinal value of $P$. sibiricum, its studies mainly focus on active ingredient and pharmacodynamics. Because of growth habit of $P$. sibiricum, it grows mostly in worse conditions. The special growth condition gives P. sibiricum strong ability to resist some abiotic stresses (Jo et al., 2017; Zhao et al., 2018; Pan et al., 2020). To date, however, studies on molecular basis of resistance to abiotic stresses in P. sibiricum are extremely rare. In this study, a novel WRKY TF gene from $P$. sibiricum was isolated and identified for the first time. Due to numbers of conserved WRKY domain, the WRKY proteins in plants are mainly classified into three groups with I, II, and III groups. Members of group I include two WRKY domains and one or two $\mathrm{C}_{2} \mathrm{H}_{2}$ zinc-finger sequences, while Group II contains a WRKY-domain along with a zincfinger $\left(\mathrm{C}_{2} \mathrm{H}_{2}\right)$ sequence (Eulgem et al., 2000). Furthemore, the WRKY domain also presents in group III but contained a $\mathrm{C}_{2} \mathrm{HC}$ type zinc-finger sequence. In this study, it is showed that PsWRKY1 belongs to group I because of two WRKY domains and two $\mathrm{C}_{2} \mathrm{H}_{2}$ zinc-finger sequence.

It has been indicated that the WRKY TFs are involved in some abiotic stress responses. However, they probably have different function in various plants even in same plant. For example, OsWRKY74 from rice is involved in tolerance to cold stress (Nuruzzaman et al., 2014), but OsWRKY30 can enhance resistance to drought stress. VpWRKY1, $V p W R K Y 2$, and $V p W R K Y 3$ from $V$. pseudoreticulata all are involved in salt stress, while $V p W R K Y 2$ can enhance resistance to cold stress (Srivastava et al., 2018). TaWRKY2 from wheat can enhance tolerance to drought and salt stresses, while TaWRKY19 not only improves resistance to salt and drought, but also enhances cold tolerance. Similarly, GmWRKY27 from soybean can improve resistance to cold stress, but not resistance to drought or salt stresses, whereas GmWRKY54 exhibit more tolerance to drought and salt stresses (Song et al., 2016). In this study, the transcriptional levels of PsWRKY1 gene are induced by low temperature and drought stresses but not by salt stress, which showed that PsWRKYl might enhance tolerance to low temperature and drought stresses.

It is well known that abiotic stress in plants are related to a complicated antioxidant defense system for ROS scavenging. Many ROS-scavenging enzymes such as POD, CAT, SOD, and non-enzymatic antioxidants can impact various physiological and biochemical processes (Banerjee et al., 2015). Previous studies showed that the antioxidant-related genes are closely related to WRKY TFs. CaWRKY4O in pepper can increase the expression of NtAPX, NtSOD1, NtGST1, and NtCAT1, enhancing high temperature tolerance in tobacco (Zhang et al., 2019). Some GhWRKY genes from cotton can improve the resistance to salt stress by reducing the MDA content and increasing antioxidant activities. GmWRKY27 from soybeans can improve resistance to the salt and drought stresses by modulating peroxidase genes (Song et al., 2016). ZmWRKY106 from Zea may can reduce ROS content and increase the activities of SOD, POD and CAT under drought treatment (Zhang et al., 2019). OsWRKY76 in rice increases the expression of antioxidant enzymes related genes, such as peroxidases and GST (OsGSTU5), thereby enhancing resistance to cold stress (Yokotani et al., 2013). WRKY44 in wheat also enhances drought 
and salt tolerance by increasing soluble sugar and proline contents and SOD, CAT, and POD activities (Satapathy et al., 2018). According to what mentioned above, when SOD activity and proline content increase but MDA levels and relative electrolyte leakage reduce, the abiotic stress resistance of plants improves more. Here, it was showed that SOD activity and proline content all increased under cold and drought stresses in overexpression Arabidopsis, whereas MDA levels and relative electrolyte leakage reduced under same stresses.

\section{Conclusion}

$P$. sibiricum has very strong ability of abiotic resistance because of the special growth conditions. We identified a novel WRKY gene $P s W R K Y 1$ in $P$. sibiricum for the first time. Sequence and evolutionary analysis showed that PsWRKY1 has the typical domains of WRKY protein, which is most closely related to the WRKY protein from E. grandis. Gene expression analysis indicated that expression of PsWRKYI was induced by drought and cold stresses. Characterization of PsWRKYI in transgenic Arabidopsis indicated that PsWRKYl enhanced the drought and cold resistance. These results indicated the functions of PsWRKY1 in response to cold and drought stresses in $P$. sibiricum, which could be utilized in Polygonatum plants improvement program to develop cold and drought tolerance variety for getting high yields. The cold and drought tolerances in plants are the complex quantitative trait. It is not enough to assess the cold and drought tolerance ability of $P$. sibiricum only based on identification of PsWRKYI in Arabidopsis. The specific regulatory relationship among $P S W R K Y 1$ and other impact factors in $P$. sibiricum still requires further investigation.

Acknowledgments. We express our gratitude to the anonymous reviewers for helpful comments to improve the manuscript. This work was supported by the Promotion and Demonstration Project of Forestry Science and Technology from Central Finance (2022TG16), Liaoning Revitalization Talents Program (XLYC1902081) and Project of Task for Discipline Construction in Liaoning Academy of Agricultural Sciences (2019DD217032).

\section{REFERENCES}

[1] Amorim, L. L. B., da Fonseca, R., Neto, J. P. B., Guida-Santos, M., Crovella, S., BenkoIseppon, A. M. (2017): Transcription Factors Involved in Plant Resistance to Pathogens. Curr. Protein Pept. Sci. 18(4): 335-351.

[2] Banerjee, A., Roychoudhury, A. (2015): WRKY proteins: Signaling and regulation of expression during abiotic stress responses. - World J. Sci. 2015: 807560.

[3] Del-Rio, D., Stewart, A. J., Pellegrini, N. (2005): A review of recent studies on malondialdehyde as toxic molecule and biological marker of oxidative stress. - Nutr. Metab Cardiovasc Dis. Sci. 15(4): 316-28.

[4] Eulgem, T., Rushton, P. J., Robatzek, S., Somssich, I. E. (2000): The WRKY superfamily of plant transcription factors. - Trends. Plant Sci. 5: 199-206.

[5] Gong, X., Zhang, J., Hu, J., Wang, W., Wu, H., Zhang, Q., Liu, J. (2015): FcWRKY70, a WRKY protein of Fortunella crassifolia, functions in drought tolerance and modulates putrescine synthesis by regulating arginine decarboxylase gene. - Plant Cell Environ. Sci. 38(11): 2248-2262.

[6] Jiang, J., Ma, S., Ye, N., Jiang, M., Cao, J., Zhang, J. (2017): WRKY transcription factors in plant responses to stresses. - J. Integr Plant Biol. Sci. 59(2): 86-101. 
[7] Jo, K., Jeon, S., Ahn, C. W., Han, S. H., Suh, H. J. (2017): Changes in Drosophila melanogaster Sleep-Wake Behavior Due to Lotus (Nelumbo nucifera) Seed and Hwang Jeong (Polygonatum sibiricum) Extracts. - Prev. Nutr. Food Sci. 22(4): 293-299.

[8] Kiranmai, K., Lokanadha, R. G., Pandurangaiah, M., Nareshkumar, A., Reddy, V., Lokesh, U., Venkatesh, B., Johnson, A., Sudhakar, C. (2018): A Novel WRKY Transcription Factor, MuWRKY3 (Macrotyloma uniflorum Lam. Verdc.) Enhances Drought Stress Tolerance in Transgenic Groundnut (Arachis hypogaea L.). - Plants. Front. Plant Sci. 9: 346.

[9] Liu, G. J., Liu, M. K., Xu, Z. R., Yan, X. F., Wei, Z. G., Yang, C. P. (2009): Construction and analysis of a forward and reverse subtractive cDNA library from leaves and stem of Polygonum sibiricum Laxm. under salt stress. - Yi Chuan Sci. 31(4): 426-33.

[10] Liu, Y., Yang, T., Lin, Z., Gu, B., Xing, C., Zhao, L., Dong, H., Gao, J., Xie, Z., Zhang, S., Huang, X. (2019): A WRKY transcription factor PbrWRKY53 from Pyrus betulaefolia is involved in drought tolerance and AsA accumulation. - Plant. Biotechnol. J. Sci. 17(9): 1770-1787.

[11] Luo, X., Bai, X., Sun, X., Zhu, D., Liu, B., Ji, W., Cai, H., Cao, L., Wu, J., Hu, M., Liu, X., Tang, L., Zhu, Y. (2013): Expression of wild soybean WRKY20 in Arabidopsis enhances drought tolerance and regulates ABA signalling. - J. Exp Bot. Sci. 64(8): 2155-21691.

[12] Luo, D. L., Ba, L. J., Shan, W., Kuang, J. F., Lu, W. J., Chen, J. Y. (2017): Involvement of WRKY Transcription Factors in Abscisic-Acid-Induced Cold Tolerance of Banana Fruit. - J. Agric Food Chem. Sci. 65(18): 3627-3635.

[13] Mitsuda, N., Ohme-Takagi, M. (2009): Functional analysis of transcription factors in Arabidopsis. - Plant Cell Physiol. Sci. 50: 1232-1248.

[14] Ng, D. W., Abeysinghe, J. K., Kamali, M. (2018): Regulating the Regulators: The Control of Transcription Factors in Plant Defense Signaling. - Int. J Mol. Sci. 19(12): 3737.

[15] Nuruzzaman, M., Sharoni, A. M., Satoh, K., Kumar, A., Leung, H., Kikuchi, S. (2014): Comparative transcriptome profiles of the WRKY gene family under control, hormonetreated, and drought conditions in near-isogenic rice lines reveal differential, tissue specific gene activation. - J. Plant Physiol. Sci. 171(1): 2-13.

[16] Pan, J., Lu, W., Chen, S., Cao, T., Chi, L., He, F. (2020): Characterization of the complete chloroplast genome of Polygonatum sibiricum (Liliaceae), a well-known herb to China. Mitochondrial. DNA B Resour. Sci. 5(1): 528-529.

[17] Qu, C. P., Xu, Z. R., Liu, G. J., Liu, C., Li, Y., Wei, Z. G., Liu, G. F. (2010): Differential expression of copper-zinc superoxide dismutase gene of Polygonum sibiricum leaves, stems and underground stems, subjected to high-salt stress. - Int. J. Mol. Sci. 11(12): 523445 .

[18] Rushton, D. L., Tripathi, P., Rabara, R. C., Lin, J., Ringler, P., Boken, A. K., Langum, T. J., Smidt, L., Boomsma, D. D., Emme, N. J., Chen, X., Finer, J. J., Shen, Q. J., Rushton, P. J. (2012): WRKY transcription factors: Key components in abscisic acid signalling. - Plant Biotechnol. J. Sci. 10: 2-11.

[19] Satapathy, L., Kumar, D., Kumar, M., Mukhopadhyay, K. (2018): Functional and DNAprotein binding studies of WRKY transcription factors and their expression analysis in response to biotic and abiotic stress in wheat (Triticum aestivum L.). - 3 Biotech. Sci. 8(1): 40.

[20] Song, H., Wang, P., Hou, L., Zhao, S. Z., Zhao, C. Z., Xia, H., Li, P., Zhang, Y., Bian, X. T., Wang, X. J. (2016): Global Analysis of WRKY Genes and Their Response to Dehydration and Salt Stress in Soybean. - Front. Plant Sci. 7: 9.

[21] Srivastava, R., Kumar, S., Kobayashi, Y., Kusunoki, K., Tripathi, P., Kobayashi, Y., Koyama, H., Sahoo, L. (2018): Comparative genome-wide analysis of WRKY transcription factors in two Asian legume crops: Adzuki bean and Mung bean. - Rep. Sci. 8(1): 16971.

[22] Sun, X., Zhang, L., Wong, D. C. J., Wang, Y., Zhu, Z. F., Xu, G. Z., Wang, Q. F., Li, S. H., Liang, Z. C., Xin, H. P. (2019): The ethylene response factor VaERF092 from Amur 
grape regulates the transcription factor VaWRKY33, improving cold tolerance. - Plant J. Sci. 99(5): 988-1002.

[23] Wei, Z. P., Ye, J. F., Zhou, Z. Q., Chen, G., Meng, F. J., Liu, Y. F. (2021): Isolation and characterization of PoWRKY, an abiotic stress-related WRKY transcription factor from Polygonatum odoratum. - Physiol Mol Biol Plants 27: 1-9.

[24] Yokotani, N., Sato, Y., Tanabe, S., Chujo, T., Shimizu, T., Okada, K., Yamane, H., Shimono, M., Sugano, S., Takatsuji, H., Kaku, H., Minami, E., Nishizawa, Y. (2013): WRKY76 is a rice transcriptional repressor playing opposite roles in blast disease resistance and cold stress tolerance. - J. Exp Bot. Sci. 64(16): 5085-5097.

[25] Zhang, Y., Yu, H., Yang, X., Li, Q., Ling, J., Wang, H., Gu, X., Huang, S., Jiang, W. (2016): CsWRKY46, a WRKY transcription factor from cucumber, confers cold resistance in transgenic-plant by regulating a set of cold-stress responsive genes in an ABA-dependent manner. - Plant Physiol. Biochem. Sci. 108: 478-487.

[26] Zhang, L., Zhao, T., Sun, X., Wang, Y., Du, C., Zhu, Z., Gichuki, D. K., Wang, Q. F., Li, S. H., Xin, H. P. (2019): Overexpression of VaWRKY12, a transcription factor from Vitis amurensis with increased nuclear localization under low temperature, enhances cold tolerance of plants. - Plant. Mol Biol. Sci. 100(1-2): 95-110.

[27] Zhao, P., Zhao, C., Li, X., Gao, Q. Z., Huang, L. Q., Xiao, P. G., Gao, W. Y. (2018): The genus Polygonatum: A review of ethnopharmacology, phytochemistry and pharmacology. - J. Ethnopharmacol. Sci. 214: 274-291.

\section{APPENDIX}

\section{Supplementary material}

Table S1. Details of primers used in this study

\begin{tabular}{|c|c|c|c|}
\hline Name & Forward Primer $\left(5^{\prime}-3^{\prime}\right)$ & Reverse Primer (5'-3') & $\begin{array}{c}\text { Involved in the } \\
\text { process }\end{array}$ \\
\hline WRKY-C & $\begin{array}{c}\text { GGTC(A/G)AAA }(\mathrm{G} / \mathrm{A} / \mathrm{C}) \mathrm{AC}(\mathrm{G} / \mathrm{A}) \\
\mathrm{GT}(\mathrm{G} / \mathrm{T}) \mathrm{AA}(\mathrm{G} / \mathrm{A}) \mathrm{GGGA}\end{array}$ & $\begin{array}{c}\text { TAC }(\mathrm{C} / \mathrm{T}) \mathrm{TT}(\mathrm{C} / \mathrm{T}) \mathrm{TGCCC}(\mathrm{A} / \mathrm{G}) \mathrm{T} \\
\mathrm{A}(\mathrm{T} / \mathrm{C}) \mathrm{TTTC}\end{array}$ & $\begin{array}{c}\text { Cloning of the } \\
\text { conserved sequences } \\
\text { of WRKY genes }\end{array}$ \\
\hline $5 \mathrm{P} 1$ & --- & \begin{tabular}{|c|} 
ACCTCTCGACCTTCTTCTTGG \\
TC \\
\end{tabular} & 5'RACE \\
\hline $5 \mathrm{P} 2$ & --- & TACGTGCACTTGTAGTAGCTC & \\
\hline $5 \mathrm{P} 3$ & --- & CGTCTTTTGACCATATTCCTCC & \\
\hline $3 \mathrm{P} 1$ & GATATTCTTGACGATGGATATA & --- & 3'RACE \\
\hline $3 \mathrm{P} 2$ & GAAAATACGGGCAGAAAGTA & --- & \\
\hline$\beta$-actin & CGGGGAAACTTACCAGGTCC & TCCACCAACTAAGAACGGCC & $\begin{array}{l}\text { Primers of the } \\
\text { reference gene for } \\
\text { qRT-PCR }\end{array}$ \\
\hline $\begin{array}{c}\text { WRKY- } \\
\text { qRT }\end{array}$ & CGAAAACAGCAACATGGGCA & TCTTCTTGGTCGGGCAGTTC & $\begin{array}{c}\text { Expression analysis } \\
\text { by qRT-PCR }\end{array}$ \\
\hline $\begin{array}{l}\text { WRKY- } \\
\text { OE }\end{array}$ & $\begin{array}{c}\text { GGACTAGTATGGCTTCTTCAAC } \\
\text { TGGGA }\end{array}$ & $\begin{array}{c}\text { GGGGTACCTCAACATAGCAG } \\
\text { CGATTCG }\end{array}$ & $\begin{array}{c}\text { Construction of } \\
\text { eukaryotic expression } \\
\text { vector for over- } \\
\text { expressing }\end{array}$ \\
\hline
\end{tabular}


Table S2. The submitted sequences of WRKY transcription factors with Genbank accession No. for construction of phylogenetic tree

\begin{tabular}{|c|c|c|c|c|}
\hline Gene name & \begin{tabular}{|c|} 
Genbank \\
accession No.
\end{tabular} & $\begin{array}{c}\text { Size of putative } \\
\text { protein (residues) }\end{array}$ & $\begin{array}{c}\text { Level of similarity with } \\
\text { PsWRKY1 (\%) }\end{array}$ & Plant source \\
\hline PsWRKY1 & MK256764 & 510 & 100 & Polygonatum sibiricum \\
\hline AcWRKY 24 & XP_020101951 & 537 & 54.46 & Ananas comosus \\
\hline AoWRKY24 & XP_020271607 & 487 & 72.88 & Asparagus officinalis \\
\hline CcWRKY24 & XM_020356797 & 523 & 54.22 & Cajanus cajan \\
\hline CsWRKY24 & XP_028051918 & 557 & 42.22 & Camellia sinensis \\
\hline CarWRKY26 & XP_004500327 & 502 & 36.75 & Cicer arietinum \\
\hline CeWRKY24 & XP_027175580 & 565 & 40.88 & Coffea eugenioides \\
\hline CmWRKY24 & XP_022964929 & 559 & 39.89 & Cucurbita moschata \\
\hline DoWRKY & AHF55741 & 542 & 44.44 & Dendrobium officinale \\
\hline EgWRKY 24 & XP_019706087 & 570 & 47.58 & Elaeis guineensis \\
\hline EgrWRKY26 & XP_018724852 & 549 & 40.31 & Eucalyptus grandis \\
\hline LrWRKY33 & ART33472 & 549 & 47.86 & Lilium regale \\
\hline LaWRKY 24 & XP_019418126 & 535 & 37.04 & Lupinus angustifolius \\
\hline McWRKY24 & XP_022154851 & 424 & 32.19 & Momordica charantia \\
\hline MaWRKY 24 & XP_009383300 & 519 & 41.88 & Musa acuminata \\
\hline PhWRKY24 & XP_025819311 & 552 & 36.61 & Panicum hallii \\
\hline PeWRKY26 & XP_020596685 & 554 & 43.02 & Phalaenopsis equestris \\
\hline PdWRKY24 & XP_026663206 & 549 & 45.73 & Phoenix dactylifera \\
\hline SiWRKY25 & XP_011080757 & 542 & 38.32 & Sesamum indicum \\
\hline SgWRKYG & AZU90760 & 402 & 30.91 & Siraitia grosvenorii \\
\hline$S b W R K Y 24$ & XP_021310889 & 556 & 37.18 & Sorghum bicolor \\
\hline VcWRKY33 & AXR71208 & 392 & 32.05 & Vaccinium corymbosum \\
\hline $\operatorname{VrWRKY24}$ & XP_014489595 & 526 & 50.18 & Vigna radiata \\
\hline$Z m W R K Y$ & NP_001354299 & 555 & 37.75 & Zea mays \\
\hline
\end{tabular}

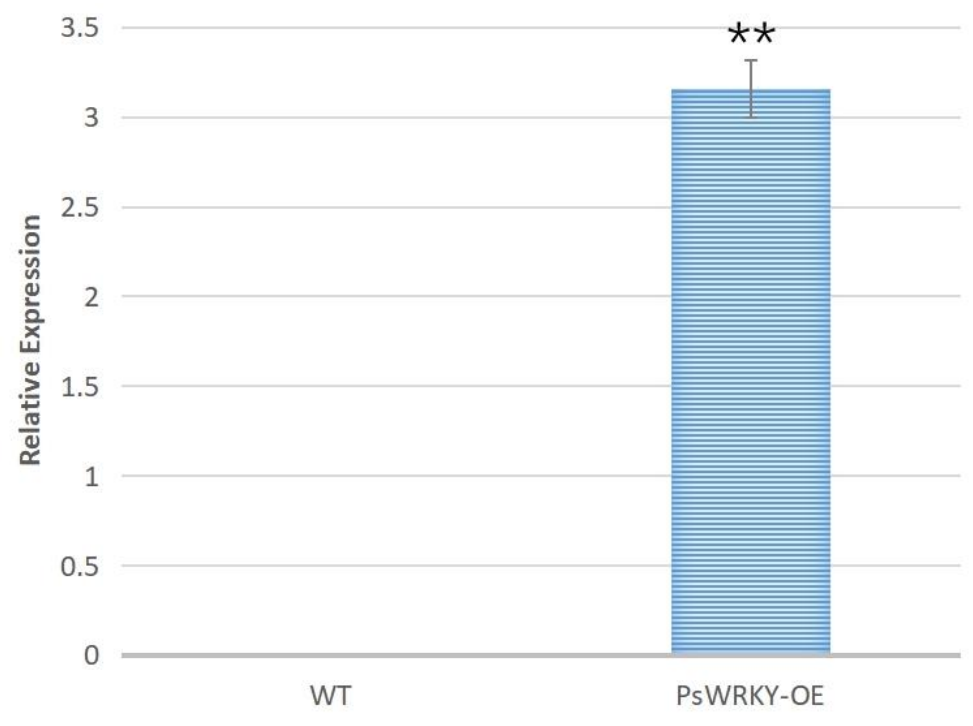

Figure S1. qRT-PCR for confirming PsWRKY1 transgenic plants. Asterisks indicate a significant difference compared to WT by Student's t test $(* * P<0.01)$ 